\title{
UNA APROXIMACIÓN MORFOLÓGICA A LAS CONSTRUCCIONES CLASIFICATORIAS EN LA LENGUA DE SIGNOS ESPAÑOLA
}

\author{
ÁNGEL HERRERO \\ Universidad de Alicante. Unidad de Investigación de Lingüística de las lenguas de signos \\ angel.herrero@ua.es
}

\begin{abstract}
Resumen
Since the pioneering work of Ted Supalla in 1978, it has been demostrated that sign languages use classifieres, specially in classifier predicates, in a typologically congruent way that seems nevertheless to exceed the limits of oral channel. In this article we review the main approaches to classifiers in sign and oral languages and provide the clasificatory system in Spanish Sign Language (LSE) with morphological criteria typologically attested. We differentiate between classificatory noums and predicate classifiers, and between introflexive classifiers and classifier prefixes
\end{abstract}

\section{Introducción}

El descubrimiento y el estudio de nuevas lenguas acarrea con frecuencia el de categorías y procedimientos lingüísticos que renuevan la imagen que teníamos de nuestras propias lenguas y que resultan valiosísimos para los estudios tipológicos. Ya en la primera edición, la de 1921, de Language. An Introduction to the Study of Speeech, Edward Sapir señalaba que

En muchos idiomas indios de Estados Unidos o del Asia Oriental, es preciso hacer constar que el objeto pertenece a cierta categoría por su forma (...) y a veces hasta hace falta decir que el objeto es o es manejado de determinada manera (Sapir, 1974: 117)

Y daba a continuación varios ejemplos de lenguas atabascanas, para concluir afirmando que "las categorías lingüísticas constituyen un dogma creado en otras épocas". En 1932, el mismo Sapir analiza en un breve pero intensísimo trabajo la aplicación metafórica de clasificadores a nombres abstractos para la creación de juegos de palabras (puns) en una lengua atabascana, el Navajo.

El primer lingüista del que tengo noticias que emprendiera un estudio sistemático de la gramática de una lengua de signos (en este caso la lengua de signos italiana) desde un punto de vista tipológico, y, como Sapir muchos años después, inspirado por el asombro ante lo nuevo, fue el español Lorenzo Hervás (1795). Su obra tipológica fue admirable, especialmente por la información de numerosas lenguas amerindias hasta entonces desconocidas; el estudio de la lengua de signos (que él creía universal) en Roma, le inspiró un gran número de ideas que inciden en la naturaleza tipológica de estas lenguas: 
No obstante de haber escrito sobre las lenguas diez tomos, de los que he publicado cinco, con el examen que he hecho de las ideas gramaticales y de la pronunciación de los sordomudos (sic), he aprendido y descubierto algunas verdades, que al escribir los diez tomos se habían ocultado a mi mente. (Hervás, 1795: 67)

El fenómeno de la clasificación, sin embargo, no alentó estudios tipológicos sistemáticos, es decir, universalistas, hasta hace tres décadas ${ }^{1}$, con los trabajos de Dixon (1968), Greenberg (1972), Allan (1977), Craig (1986), Corbett (1991), Croft (1994), Grinevald (2000), y Aikenvald (2000), entre los más destacados.

La eclosión de este interés tipológico de la lingüística por los fenómenos relacionados con la clasificación nominal coincide en el tiempo, y en cierto modo en el lugar, con los primeros estudios de morfología de la American Sign Language, que desde un principio señalaron el fenómeno de la clasificación en las lenguas de signos (en adelante LSs) como una de sus características morfológicas; así, los trabajos de Frishberg² (1975), Kegl y Willbur (1976), y sobre todo Supalla (1978), que constituyó sin duda la referencia inicial para todos los trabajos sobre clasificadores en LSs. Y es que, como ocurría en aquellas lenguas amerindias, en las lenguas de signos la clasificación es un fenómeno recurrente, con lo que se hace posible su comparación tipológica. De hecho, el ejemplo de los clasificadores verbales en el Navajo ha servido, a partir de Allan (1977) y Supalla (1978) y durante muchos años, como botón de muestra del paralelismo entre las construcciones clasificatorias de las lenguas orales o habladas (en adelante LOs) y de las LSs ${ }^{3}$.

Los estudios sobre clasificación en LSs han ido creciendo en los últimos años, y en direcciones diversas, como demuestra la dispersión terminológica (Schembri, 2003: 4) -bastante común en la lingüística de lenguas de signos-, que continúa hasta hoy (clasificadores, signos clasificadores, verbos clasificadores, predicados clasificadores, predicados polimorfémicos, signos polisintéticos, signos policomponenciales, signos productivos, etc.). Esta dispersión, que se da también, aunque en menor medida, en la tipología general, especialmente a partir de las críticas a la denominación de predicado clasificador (Croft, 994), evidencia una falta de acuerdo sobre la naturaleza lingüística del fenómeno de la clasificación nominal en las LSs., pero también demuestra el carácter central que esta cuestión parece tener en relación con la congruencia tipológica de estas lenguas con las lenguas orales. $Y$ es que en la misma medida en que la clasificación se inserta en un punto de vista tipológico, ha surgido (o resurgido, pues que se trata de una tentación recurrente) la cuestión de que en las LSs se emplee un segundo modo de representación visual que tendrían los signantes ${ }^{4}$, además del lingüístico, modo en el que la simultaneidad (también morfológica) y la iconicidad aso-

Aikenvald (2000: 5) señala que el primer panorama descriptivo, sobre clasificación, género y clasificadores numerales, lo aportó Royen (1929), y uno de los primeros en realizar una comparación del empleo de clasificaciones en dos familial lingüísticas, Worsley (1954).

2 Nancy Frishberg había publicado en 1972 un trabajo sobre la clasificación en el Navajo (Frishberg, 1972)

3 Sobre la crítica posterior a este paralelismo entre los clasificadores verbales del Navajo y la ASL, ver CogillKoez (2000b), y Schembri (2003: 17).

4 Lorenzo Hervás (1795: 66) "La gramática de los Sordomudos es totalmente mental, y la nuestra es mental y verbal". Ver la defensa que hace del carácter libre del género o del tiempo, y la idea de la unión de nombre a nombre para expresar relaciones en otras lenguas más gramaticalizadas, en 76 y ss, así como la observación de que en algunas lenguas, entre ellas las LSs, las preposiciones "se deberían Hlamar posposiciones"(95); en II: 147, para describir los signos de las preposiciones, Hervás hace uso de CLs avant la lettre. 
ciadas a los clasificadores podrían tener justificación (Liddel, 2003; Cogil-Koez, 2000a.). Desde nuestro punto de vista, y como iremos señalando en este trabajo, ese segundo modo de representación, responsable de la extraordinaria plasticidad de los signos, se basa en el modo lingüístico, y es éste el que aquí vamos a considerar.

Desde el punto de vista de la morfología de la clasificación nominal en la LSE, creemos que con este trabajo realizamos dos aportaciones específicas: el reconocimiento de un tipo de clasificador, los nombres clasificatorios o clasificadores nominales, que no han sido aún apenas estudiados en las $\mathrm{LSs}^{5}$, y el de un tipo de morfología de clasificación, las más usual en las LOs, como es la afijal, que tampoco se ha planteado hasta ahora dentro de los estudios de clasificadores en las LSs, sobre todo por el modelo fonológico dominante, que considera el signo como un fenómeno simultáneo o de secuencia sostenido-movimiento pero sin estructura silábica. La propuesta de tipos de clasificadores en la LSE que vamos a realizar viene a ser en este sentido una prueba del modelo de sílaba que hemos planteado como justificación de la escritura de la LSE (Herrero, 2004).

\section{Los tipos de clasificador}

Unos de los aspectos que han evolucionado en los estudios de tipología relativos a la clasificación, concretamente desde Allan (1977) hasta Aikhenvald (2000), es precisamente el de la propia clasificación de clasificadores, estrechamente vinculada a la identificación misma del fenómeno como fenómeno morfológico. En las lenguas encontramos muchos fenómenos de clasificación (en el léxico y en la gramática), pero cuando hablamos de un clasificador estamos refiriéndonos a un morfema dentro de una construcción ${ }^{6}$, que podemos llamar por eso construcción clasificatoria, y que tiene los requisitos de ser abierto (overt), es decir, identificable, de formar parte de un subsistema gramatical que está semánticamente motivado por los nombres sobre los que incide o sustituye, de actuar anafóricamente respecto a estos nombres, y de poderse suprimir o cambiar por razones discursivas o pragmáticas (Grinevald, 1996; Aikhenvald, 2000).

Nuestro objetivo es estudiar la variedad morfológica con que se realizan esos morfemas overt, pero por razones metodológicas vamos a detenernos antes en la clasificación tipológica, funcional, muy importante a la hora de valorar los estudios sobre clasificadores en lenguas de signos. Así, de los cuatro tipos propuestos por Allan en 1977 (numeral classifiers, concordial classifiers, predicate classifiers, intralocative classifiers), se pasó en el 2000 a una clasificación más compleja, la de Grinevald y Aikhenvald (noum classifiers, numeral classifiers, possesive or genitive classifiers, verbal classifiers, locative classifieres and deictic classifiers). Los cuatro primeros consituyen para Grinevald (2000) las cuatro clases principales de clasificador; las dos últimas son clases menores.

Si comparamos las dos clasificaciones se observa por un lado la desaparición de los concordial classifiers, considerados como clases nominales (como el género). La diferencia metodológica entre clases nominales y clasificadores la planteó por primera vez Dixon (1986), estableciendo una correlación entre tipo lingüístico y sistema de clasificación, y planteando la diferencia de gramaticalización que se observa entre clases nominales y clasificadores: las 
primeros constituyen clases reducidas y cerradas, los segundos clases amplias y abiertas; las primeros son propios de lenguas fusivas o aglutinantes, los segundos de lenguas aislantes. Pero lo más notable del cambio está en la incorporación del noum classifier.

Frente al resto de clasificadores mayores (numerales, posesivos, verbales) y menores (locativos, demostrativos), el clasificador nominal o nombre clasificatorio ${ }^{7}$ es prototípicamente un morfema libre, un nombre, normalmente antepuesto al núcleo, con el que tiene frecuentemente una relación de genérico/específico, y al que acompaña pero con el que también puede anaforizarse o reducirse, actuando uno u otro como proforma en función del discurso y el contexto (Aikhenvald, 2000: 86). Pero los demás tipos son prototípicamente morfemas ligados a la categoría respectiva (al adjetivo, al verbo, al locativo, etc). El clasificador numeral presenta ambas formas. Si imaginamos el fenómeno de la clasificación dentro de una escala de gramaticalización del léxico, el nombre clasificatorio ocuparía los primeros rangos, muy cerca aún de la condición léxica del nombre.

Un nombre clasificatorio es, como hemos dicho, un nombre que se puede emplear como nombre común pero además se emplea como clasificador, es decir que se selecciona por razones semánticas (para categorizar a $\mathrm{N}$ ), completa el significado del nombre y no es extensivo a todos los nombres ni siempre obligatorio, pero puede actuar como proforma anafórica de la construcción. Por ejemplo, en el Jacalteco (maya), Juan vio una culebra, se expresa

$$
\text { (1) xil naj wuwan no7 lab'a }
$$

literalmente vió hombre-Juan animal-culebra. Pero esta oración puede ser reducida anafóricamente en:

(2) xil naj wuwan no7

literalmente vió hombre-Juan animal, pero que debe entenderse como Juan la vió (a la culebra referida).

Un mismo nombre puede venir acompañado de distintos clasificadores. Por ejemplo, en la lengua Minangkabau, de Oceanía, se distingue el limón (como fruto) buah limau (literalmente fruto limón) de limonero batang limau (literalmente árbol limón). Su gramaticalización puede llevar a formas clíticas, o a convertirlo en procedimiento de derivación (Aikenvald, 2000: 91-93), como parece ser el caso de lenguas amerindias arawakianas y tucanas. El número de nombres clasificatorios oscila. Por ejemplo, la lengua Yidiny, australiana, tiene un conjunto de 20 nombres clasificatorios que se anteponen a los nombres para clasificarlos. hombre, mujer, persona, pájaro, rana, hormiga, árbol, vino, fuego, piedra, tierra, lanza, saco, canoa, carne (comestible), vegetal (comestible), bebida, hábitat, movimiento, y ruido intencional. El akateh, lengua maya, tiene 14 nombres clasificatorios, que son obligatorios. En otras lenguas se han datado hasta un centenar. Hay por lo tanto lenguas que parecen tener un número cerrado de nombres de este tipo, y lenguas en las que la investigación parece

7 Todos los clasificadores, libres o ligados, son clasificadores de nombres, es decir, clasificadores nominales; por ello creemos más correcta la denominación de nombre clasificatorio; en el resto de clasificadores (clasificadores numerales, posesivos, verbales, etc...) se entenderán estos adjetivos (numerales, verbales, etc.) como indicadores de la categoria en la que se produce el clasificador: clasificadores que se dan en (o con) el número, los posesivos, etc... 
apuntar a paradigmas aún abiertos, o a nombres clasificatorios más difíciles de detectar por su coincidencia con otras construcciones nominales.

El estudio de los clasificadores en las LSs no se ha detenido apenas hasta ahora en este tipo de clasificador, el nombre clasificatorio, limitándose exclusivamente a los clasificadores predicativos (Aikhenvald, 2003: 89). La condición de morfema libre (o lexema gramaticalizado), y la posible confusión de la construcción clasificatoria de nombre clasificatorio con la complementación nominal, la composición o la derivación pueden explicar en parte esta omisión. Pero nuestra investigación nos ha llevado a la conclusión de que la LSE sí tiene nombres clasificatorios.

Como en este trabajo pretendemos abordar el fenómeno de la clasificación en la LSE desde el punto de vista de la morfología, podemos empezar distinguiendo dos tipos de clasificador claramente diferentes desde el punto de vista morfológico:

(a) Los Nombres clasificatorios, que son morfemas libres o palabras-signos independientes, para delimitar el ámbito de significación del nombre, como en el signo río (AGUA CAUCE), donde el núcleo CAUCE se clasifica con el signo AGUA.

(b) Los clasificadores, o configuraciones manuales clasificatorias, que son morfemas ligados a otra palabra-signo, es decir, incorporados a esa palabra-signo como configuración de la mano pasiva o activa (flexivos o introflexivos); por ejemplo, la configuración de palma abierta y dedos extendidos y juntos, escrita en S.E.A. (Herrero, 2003) o, para representar objetos de dos dimensiones, como al clasificar el PAPEL; o la configuración de dedos en garra, escrita en S.E.A. ó, para representar objetos de tres dimensiones, como al clasificar una ROCA. Estos clasificadores se dan siempre en otro signo no nominal, ya sea adjetivo -como en El libro grueso-, locativo -El libro de encima de la mesa- o verbo -dar un libro, mirar el libro-. En todos estos casos el clasificador (que se produce en el adjetivo grueso, en el locativo encima de, en el verbo dar y en el objeto libro) tiene como referencia el signo nominal LIBRO, y se integra en esos signos como morfema ligado ocupando el lugar fonológico de la configuración del signo.

La primera propuesta que presentamos a continuación es la de la existencia de signos con valor de nombres clasificatorios en la LSE. Posteriormente nos referiremos al resto de clasificadores, en la LSE todos configuracionales (de ahí la expresión, que preferimos, de configuración clasificatoria) y por tanto morfológicamente ligados, aunque distinguiremos entre clasificadores introflexivos o supletivos, y afijales.

\section{Nombres clasificatorios en la LSE}

En la LSE, algunos nombres que tienen que ver con el agua anteponen el signo AGUA a otro signo, para juntos producir el significado léxico. AGUA es un nombre clasificatorio en rio (AGUA CAUCE), pozo (AGUA POLEA), fuente (AGUA SURTIDOR), grifo (AGUA LLAVE), botella de agua (embotellada) (AGUA BOTELLA), tuberia (AGUA TUBO), gota (AGUA GOTA) etc.

Este uso del signo AGUA corresponde al de un nombre clasificador; volviendo a la definición anterior, se selecciona por razones semánticas (para categorizar a N), completa 
el significado del nombre y no es extensivo a todos los nombres ni siempre obligatorio (Aikhenvald, 2000: 91), pero puede actuar como proforma anafórica de la construcción. Por ejemplo, un grifo que no cesa de correr se signaría AGUA LLAVE AGUA+correr, pero en un contexto conveniente podríamos signar también LLAVE AGUA+correr. El signo AGUA está categorizando a LLAVE, y en este sentido compite con TELEVISION LLAVE, LAVADORA LLAVE, etc; pero no es extensivo, no es del todo previsible, como se ve en expresiones como AGUA MONTAÑA, AGUA COHE, o AGUA MOTOR, que nadie interpretaría como montaña de agua, coche de agua, o motor de agua, sino como agua de la montaña, agua del coche, agua del motor.

Hasta donde hemos podido investigar, en la LSE actúan como clasificatorios, además del signo AGUA, los signos nominales de PERSONA (revisor, embajador), ARBOL (manzano, naranjo), PIEDRA (mojón, empedrado), DINERO (billete, cartera) COCHE (garaje, gasolina), pero sin duda hay más. La cuestión está en cómo podemos descubrirlos, y por qué podemos afirmar que en los ejemplos anteriores estamos ante un nombre clasificatorio.

Un mismo signo, como AGUA o PERSONA, puede figurar en construcciones distintas. Por ejemplo el signo AGUA puede funcionar como

(a) nombre común núcleo, como en agua fría (AGUA FRIA)

(b) nombre común formando parte de un compuesto, como en barro (AGUA TIERRA)

(c) como nombre clasificatorio, como en pozo (AGUA POLEA).

El signo PERSONA puede funcionar como

(a) nombre común núcleo, como en persona interesante (PERSONA INTERESANTE)

(b) derivativo, para crear adjetivos a partir de nombres como beato (PERSONA PIEDAD), gentilicios como madrileño (PERSONA MADRID), o para crear nombres a partir de verbos, por ejemplo anfitrión (PERSONA INVITAR)

(c) nombre clasificatorio, como en conserje (PERSONA GALON)

Vamos a comparar a continuación el funcionamiento de los NCL con el de las tres funciones en las que podría aparecer en estas otras construcciones: la construcción de Nombre+complemento, las palabras compuestas de $\mathrm{N}+\mathrm{N}$, y los derivados nominales también de $\mathrm{N}+\mathrm{N}$.

Los NCL se colocan en la LSE, como en la mayoría de las lenguas con nombres clasificatorios, delante de $\mathrm{N}$, pero esto no es un criterio suficiente para diferenciarlos de las de su empleo como complemento, porque algunos complementos ${ }^{8}$ en la LSE se anteponen. Tampoco esa posición les diferencia en la LSE de los derivados (PERSONA MADRID).

Las propiedades que diferencian la construcción clasificatoria nominal de la construcción complementaria, de derivados y compuestos, son las tres siguientes:

(a) ser suprimible, a diferencia de las otras tres construcciones (el núcleo de la construcción complementaria, de la derivación o la composición). En este sentido funciona como un complemento en colocación.

(b) no ser separable del núcleo, como los compuestos y derivados.

(c) ser productivo, es decir, poderse aplicar a nuevos nombres, como la derivación.

8 Según nuestras investigaciones, los que indican posesión natural o permanente, relación parte/todo, y contenido, tienden a anteponer el complemento. El resto (origen, materia, función) posponen el complemento. Estas investigaciones se publicarán dentro de la Gramática visual de la LSE que estamos preparando. 
Si el nombre clasificatorio es suprimible, evidentemente no constituye el núcleo de la construcción. En efecto, si signamos una oración con nombre clasificatorio como

(3) AGUA SURTIDOR / GRANDE

o una expresión con nombre complementario, como

(4) MESA MADERA / GRANDE o AGUA MONTAÑA / BUENA

las podemos reducir respectivamente a

(5) SURTIDOR/GRANDE

MESA / GRANDE

AGUA BUENA

Pero si signamos

(6) AGUA / GRANDE

MADERA / GRANDE MONTAÑA / BUENA

el significado cambia o es absurdo. Esto demuestra que AGUA no es el núcleo, sino que lo es SURTIDOR, como lo es MESA en MESA MADERA, o AGUA en AGUA MONTAÑA. La misma posibilidad de supresión se daría en oraciones completivas.

Las otras dos construcciones que comparamos con la del nombre clasificatorio, esto es, la construcción de $\mathrm{N}+\mathrm{N}$ compuestos y de $\mathrm{N}+\mathrm{N}$ derivados, tampoco admiten supresión; pero a diferencia las construcciones complementarias comparten con la clasificatoria la segunda característica, la de no separabilidad. Entre un nombre y su complemento podemos insertar otros complementos, un demostrativo por ejemplo, pero esto no es posible si los dos nombres de la construcción forman un compuesto, o uno de ellos es un clasificador o un derivador. Además, mientras que la derivación es un mecanismo productivo, como la clasificación, la composición no lo es.

Los nombres compuestos son construcciones léxicas que deben figurar en el diccionario, como las palabras simples. Los signos sábado, domingo y fin de semana son palabras, entradas del diccionario, limitadas. Por ejemplo, en la LSE SÁBADO-DOMINGO es una palabra compuesta, pero VIERNES-SÁBADO no, ni DOMINGO-LUNES tampoco. En cambio, el sistema de clasificación nominal es un sistema abierto, que puede aplicarse a más elementos; por ejemplo, el nombre clasificatorio AGUA se puede aplicar a TUBO para tuberia, y también aparece en el signos de PECERA, de SED, etc. O el nombre clasificatorio PERSONA se aplica a GALON para significar conserje pero también a SOMBRERO para significar embajador, etc.

También los derivados, como los nombres clasificatorios, son productivos. Podemos aplicarlos a signos nuevos, por ejemplo una persona que viva en el pueblo de Cocentaina (un contestano). Se signaría PERSONA COCENTAINA; o un envidioso, se signaría PERSONA ENVIDIA, etc. Pero la productividad de una derivación es mucho más sistemática que la de un clasificador.

Creemos que con el test de estas propiedades, que resumimos en el cuadro siguiente, es sencillo confirmar la naturaleza clasificatoria de los nombres en la LSE. 


\begin{tabular}{|l|c|c|c|}
\hline & Supresión & Separación & Productividad \\
\hline $\mathrm{N}+$ Ncomplemento & $-/+$ & + & + \\
\hline Nclasificador $+\mathrm{N}$ & + & - & + \\
\hline Nderivador $+\mathrm{N}$ & - & - & + \\
\hline $\mathrm{N}+\mathrm{N}$ (compuesto) & - & - & - \\
\hline
\end{tabular}

Cuadro 1

\section{Los clasificadores propiamente dichos}

Del resto de clasificadores relacionados en la tipología, la LSE los presenta en construcciones verbales, adjetivales, y locativas ${ }^{9}$, aunque no posee clasificadores numerales (los más abundantes en las LOs) ni posesivos. En todos estos casos la clasificación se realiza mediante un morfema ligado, el morfema de clasificación que se expresa mediante una Q-clasificatoria que sustituye a la configuración de la mano en la forma habitual del signo verbal, adjetival o locativo correspondiente. Morfológicamente forman por consiguiente una clase diferente de la de los nombres clasificatorios, que son morfemas libres. Esta morfología, sin embargo, ha dado lugar a muy diferentes interpretaciones, como se refleja en la terminología que ya hemos citado. Las modificaciones configuracionales y los de otros componente fonológicos del signo podrían acarrear, en el caso del verbo, esto es, cuando se trata de un clasificador predicativo, $\mathrm{y}$, como ha señalado Scott Lidddell (20003: 25), hasta 25 morfemas distintos expresados mediante los fonemas de Lugar, Configuración, Orientación y Movimiento, que se flexibilizan hasta adoptar formas icónicas muy variadas. Pero evidentemente el empleo del término morfema es aquí bastante relativo o metafórico, y no lo compartimos.

Ello ha llevado a formular la hipótesis ${ }^{10}$ de que los clasificadores ( $\mathrm{y}$ esos usos figurativos del movimiento, de la orientación, etc.) son instrumentos de una representación no lingüística o no sólo lingüística sino visual. Así Karen Emmorey (2003:256) ha afirmado que "the depictive use of signing space is a gestural component of classifier costructions, rather than a morphemic component". Por eso Ted Supalla (2003:249) se pregunta. "Do classiffier predicated mark the point where ASL diverges from other human languages?" Su investigación en curso, como la nuestra en las páginas que restan, es demostrar el carácter sistemático, lingüístico, de los clasificadores, la conversión de gestos no lingüísticos en gestos lingüísticos y los procesos de gramaticalización que envuelven (2003: 256), y es en esta línea, la de deslindar lo lingüístico, en la que nosotros nos situamos.

9 En algunos libros de divulgación de la LSE se incluye también el clasificador numeral, y otros diversos (partes del cuerpo, instrumental, etc.). Estas clasificaciones combinan criterios sintácticos y semánticos, y son por ello muy borrosas. Además, confunden la clasificación y la incorporación, en el caso concreto de los numerales. Otros tipos, como los de trazo geométrico, han sido excluidos ya por otros investigadores (Wallin, 1996).

10 Como forma de iconicidad visual, la idea de dos modos de representación estaba ya planteada en Kendom (1988). 
Por ello adoptamos como referencia las características que estos clasificadores presentan en los estudios tipológicos. Pues bien, desde el punto de vista morfológico, y tomando como modelo el caso morfológicamente más complejo, el de los clasificadores verbales o predicativos, los clasificadores en las lenguas pueden aparecer ${ }^{11}$ :

(a) como nombres incorporados al V

(b) como afijos de $\mathrm{V}$

(c) y en verbos supletivos.

El primer tipo, en el que un nombre, que normalmente tiene una relación de específico a genérico con un argumento del verbo, se infija en el verbo para expresar otro argumento, lo ilustramos con el Mayali (australiana) (Aikhenvald, 2000: 150):

$$
\begin{aligned}
& \text { ga-yaw-garrm-e } \\
& 3^{a} \text { s-niño+gen-haber-presente } \\
& \text { Él tiene un niño }
\end{aligned}
$$

La incorporación, como sucede en el Mayali, es especialmente común con nombres relativos a partes del cuerpo, como en

$$
\begin{aligned}
& \text { ngan-garre-molk-bukka-ng } \\
& 3^{\mathrm{a}} \mathrm{s}+1^{\mathrm{a}} \text { obj-pantorrilla-hinchazón-enseñar-pasado } \\
& \text { Me mostró la inflamación de su pantorrilla }
\end{aligned}
$$

El segundo tipo, muy documentado en lenguas amerindias y polinésicas, consiste en la prefijación (como en lenguas atabascanas) o sufijación (como en arawakianas) del verbo por un morfema clasificador que se refiere a un nombre con función de sujeto o de objeto, caracterizándolo por algunas propiedades inherentes al mismo, como su forma. Así, en la lengua Imonda (lengua papú de Nueva Guinea), dame un coco se expresa:

$$
\begin{array}{lcc}
\text { sa } & \text { ka-a } & \text { pot-ai-hu } \\
\text { coco } & \text { a mí } & \text { Cl: FRUTO-dar-imperativo }
\end{array}
$$

Hay lenguas, como el Terêna (atabascana) que tiene varias docenas de afijos clasificatorios que caracterizan sujeto/objeto en términos de su forma, tamaño, constitución y animacidad; otras, como el Mundurukú (tupi-guaraní) cuenta con unos 100 morfemas que se refieren a la disposición o forma de los nombres. Es frecuente que el morfema clasificatorio aparezca no sólo afijado al verbo, sino al nombre al que clasifica, convirtiéndose así en una forma de concordancia sintáctica y no sólo semántica. En Mundurukú, se emplea el morfema $-b a$ clasificador de objetos largos y rígidos, en oraciones como la siguiente:
(10)

bekitkit ako-ba
niño banana-CL
o'su-ba-dobuxik
$3^{\mathrm{a}} \mathrm{s}$-Cl-encontrar 
El tercer tipo es el que ha recibido en la tipología el nombre de verbo clasificador (en lenguas amerindias y tibetanas). Sería el caso en que el clasificador que acabamos de ejemplificar no sólo representara al objeto, sino que fuera a su vez la raíz verbal. Estos verbos se seleccionan en función tanto de propiedades inherentes al nombre (forma, animacidad, etc.) como por su disposición y orientación en el espacio o la forma de llevarlas (las dos cosas están relacionadas, pues la disposición depende normalmente de la forma). Hay muchas lenguas (el inglés, el alemán, el ruso...) que tienen dobletes o tripletes léxicos para diferenciar una misma acción, por ejemplo correr, o un mismo estado, por ejemplo el estar en pie, según los sujetos a los que ataña, pero este fenómeno no es morfológico, no está paradigmáticamente abierto a otras acciones o estados. En cambio, los verbos clasificatorios son verdaderas raíces clasificatorias, seleccionadas en función de sujeto/objeto, a las que se añaden los morfemas verbales correspondientes. Así, para la raíz equivalente a estar, el Mescalero Apache (atabascana) dispone de 10 raíces concretas distintas: - 'a para objetos singulares, sólidos, redondos e inanimados; -ti para animados; -ta para alargados y rígidos, etc. En algunas lenguas, como la lengua Ika (chibchá, de Colombia), algunas raíces verbales se funden con las raíces clasificatorias y resultan parcialmente analizables (Aikhenvald, 2000: 156) como afijos. Entre al clasificador afijal y el supletivo hay pues un gradiente. No se conocen lenguas que combinen un sistema de incorporación nominal y un sistema de raíces verbales clasificatorias (Aikhenvald, 2000: 163), aunque algunas, como el Cherokee, parecen atestiguar un proceso de gramaticalización de un tipo a otro. Esta supleción morfológica analizable ha recibido a veces el nombre de morfología no concatenante (Simone, 1993: 114), o morfología introflexiva (Moreno, 1997: 33).

Parece efectivamente claro que estos tres tipos de clasificador verbal representan tres etapas, no necesariamente lineales, de gramaticalización de un fenómeno que en todos los casos es de síntesis: la de un verbo con un argumento; en el primer tipo la síntesis se produce porque el argumento se incorpora al verbo como afijo; en el segundo es un morfema, una proforma que lo clasifica, el que entra en síntesis con el verbo; en el tercero es la naturaleza o posición del argumento lo que selecciona directamente una raíz verbal sintética.

Pues bien, para determinar si en la LSE hay clasificadores verbales, y de qué tipo, es imprescindible que tengamos un modelo morfofonológico que nos permita distinguir morfemas con valor de raíz y de afijos, y su orden. Esto se hace igualmente necesario para el resto de clasificadores (adjetivales, locativos), a los que no me voy a referir sino con ejemplos.

\section{El modelo fonológico secuencial. Resumen}

Desde el inicio de la investigación moderna en lenguas de signos, a partir de los trabajos de William Stokoe, se consideró que el modo visual-gestual de estas lenguas acarreaba la simultaneidad de sus constituyentes fonológicos. De estos constituyentes, llamados parámetros (nosotros preferimos llamarlos clases de fonemas), se consideran hoy los siguientes:

a) la configuración que adopta la mano (fonemas Q)

b) el lugar donde se signa (fonemas $L$ )

c) el posible contacto de $\mathrm{Q}$ con $\mathrm{L}$ (fonemas $\mathrm{C}$ )

d) la orientación de $\mathrm{Q}$ (fonemas $\mathrm{O}$ )

e) el movimiento direccional (fonemas D)

f) el movimiento interno (fonemas F)

g) la bimanualidad (fonemas $\mathbf{S}$ ) 
La expresión no manual (cejas, boca, hombros, etc.) desempeña un papel prosódico que no vamos a tener en cuenta pues no afecta al estudio de los clasificadores. En nuestro modelo secuencial (Herrero, 2003, 2004), estos fonemas se procesan en el siguiente orden:

\section{S LCQODF}

Es decir, concebimos el signo como el desplazamiento de la mano activa (o de las dos manos, en los signos bimanuales simétricos) a un lugar $\mathrm{L}$, que es el fonema con el que arranca el signo; en ese lugar L, la mano adopta una configuración $Q$, con una orientación $\mathrm{O}$, y a continuación realiza un movimiento ya lineal (D) o interno (F) o ambos (que en este caso sí pueden ser simultáneos). En $\mathrm{S}$ escribimos pues el símbolo de la bimanualidad simétrica, pero también el de la mano pasiva cuando actúa como L de la activa.

Hay dos aspectos fundamentales de este modelo: primero, el arranque silábico en L; segundo, el carácter de núcleo silábico de Q. Ambos aspectos son fundamentales para entender la incidencia de los distintos clasificadores. Cuando la mano pasiva actúa como L, la mano activa se dirige hacia ella y adopta su $\mathrm{Q}$ correspondiente; pero la mano pasiva, a diferencia de otros L del espacio o del cuerpo (la frente, la barbilla, etc.), no es sólo un lugar, puede a su vez adoptar un L, una Q y una O propias. Esto la hace apta para la clasificación, que como estamos señalando es siempre una cuestión de configuración. En el L de estos signos, en los que se suele representar con la mano pasiva el objeto de la acción realizada por la activa, se van a producir los clasificadores afijales. En la Q de la mano activa, por otra parte, vendrán a realizarse las configuraciones clasificatorias de carácter introflexivo.

La consideración de los clasificadores afijales es muy significativa dentro de la gramática de la LSE. El orden de esta lengua, que es básicamente OV (con fenómenos de tematización muy sistemáticos), es un reflejo del orden silábico $L Q$, cuando en $L$ se ubica el clasificador del objeto (O) y, a continuación, la $\mathrm{Q}$ activa realiza un movimiento en relación a ella (V). La precedencia de la mano pasiva, como lugar de signación $\mathrm{L}$, sobre el resto de clases de fonemas se evidencia también en signos bimanuales sin clasificación, como PERDON, OBJETIVO, ENCONTRAR, etc. Hasta donde sé, esta relación entre la mano pasiva y activa y el orden OV no ha sido advertida; sin embargo, resulta absolutamente natural en la LSE y, como estamos viendo, muy significativa dentro de su gramática.

Con el sistema de escritura alfabético este orden se evidencia, esto es, visualizamos la afijación y la introflexión como lo hacemos con otras lenguas introflexivas o flexivas.

\section{Los clasificadores introflexivos en la LSE}

Como hemos venido indicando, cuando en las LSs se habla de clasificador (y al margen de los nombres clasificatorios) de hecho se está haciendo referencia una configuración, con valor morfológico ${ }^{12}$ de clasificador, que entra dentro de un signo verbal, adjetival (predicativo o no), o locativo (predicativo o no), como en los ejemplos siguientes: 
MAÑANA LIBRO DAR.CL

LIBRO Cl-ancho

LIBRO MESA Cl-encima mañana te doy el libro

libro grueso

libro encima de la mesa

Una de las características de estas configuraciones es su plasticidad, muy relacionada con la plasticidad de los mismo predicados en que se integran (verbos de ubicación, movimiento y manipulación; adjetivos de consistencia, postura, animación, tamaño y forma). O mejor, deberíamos decir con la idea de plasticidad que encierran esos verbos y adjetivos, pues significativamente son los mismos que se clasifican en las lenguas orales (Aikhenvald 2000: 296-299). Esta plasticidad se basa en la adaptación icónica de la configuración manual (y en predicados de movimiento también del fonema de movimiento), al objeto de la representación, y efectivamente, sería simultánea con lo mismo que deforma ${ }^{13}$. Esta plasticidad es muy común en el uso descriptivo o figurativo que los signantes hacen tan frecuentemente de los signos, pero creemos que se produce sobre formas sistemáticas, y que éstas pueden aislarse y estudiarse como lo que son, formas lingüísticas, aunque la adaptación icónica las convierta en elementos de una representación que ya no es lingüística sino visual.

Como ya hemos comentado, los clasificadores no son extensivos a todos los nombres, ni actúan en todos los verbos, adjetivos o locativos. Un mismo sustantivo puede admitir distintos clasificadores, según el tipo de construcción en la que entre el sustantivo (por ejemplo el signo LIBRO se puede clasificar como ò, es decir con la configuración de palma extendida y dedos juntos, y como'á, es decir con la misma configuración anterior pero con el pulgar enfrentado al resto de los dedos juntos); o puede venir clasificado o no (como el signo TELEVISION, que se clasifica en la televisión está volcada TELEVISION CL-volcarse, mediante la configuración ó, es decir, la configuración de dedos en garra, pero no en la televisión es cara). Para la clasificación de un nombre éste tiene que tener clasificadores, pero además la categoría que acoge al clasificador tiene que poder ser clasificable (como lo es el verbo volcarse, pero no el verbo pensar, por ejemplo; o el adjetivo ancho, pero no el adjetivo caro).

Sin duda hay restricciones semánticas que limitan la aplicación de los clasificadores, pero para este estudio nos interesa la forma de realización morfológica y sintáctica de estos clasificadores, que podemos contrastar con la tipología, y no su semántica.

Lo primero que quisiera señalar es la incidencia de la clasificación en adjetivos, no necesariamente predicativos, como en

\section{LIBRO $\mathrm{Cl}$-ancho GUSTAR+NO}

No me gustan los libros gruesos

También los locativos con clasificación pueden darse de forma no predicativa, como en

\section{LIBRO MESA Cl-encima AMO JUAN}

El libro de encima de la mesa es de Juan

13 Asi, Schembri (2000:8): "like morfemic palatalization or tone [Japanese, for example, where palatalization is used to indicfate 'uncontrolldness', and the bantu language, Chichewa, where the distinction between simple and recent past tense is signaled by modifications in tone] the morphemes in polymorphemic constructions mus be produced in combination with other morphemes to create a pronbunceable form. Is is thus difficult to segment the meaningful components of polimorphemic constructions" 
En segundo lugar, como ocurre en otras lenguas orales, los clasificadores pueden combinarse en la misma oración; por ejemplo al signar

\section{LIBRO CL-o'á CL-ò+ ALLI / INTERESANTE. \\ El libro delgado que está en ese estante es interesante}

En tercer lugar, en la LSE, junto a la clasificación de un nombre mediante un clasificador morfológico configuracional incorporado a verbo, adjetivo o locativo, hay otro recurso que es el de la incorporación de la configuración propia del nombre, y no la de un clasificador, a la categoria receptiva. Por ejemplo al signar

\section{TELEVISION Q(televisión)-grande}

La televisión es grande

\section{MONEDA Q(moneda)-caer}

La moneda se ha caido

\section{LAMPARA MESA Q(lámpara)-encima. \\ La lámpara está encima de la mesa}

donde a la misma configuración de TELEVISIÓN, una vez signado el signo, se aplica la extensión de brazos propia del signo GRANDE; a la configuración de MONEDA la acción de CAER y a la de LAMPARA se la ubica encima de la MESA. Sin duda, este comportamiento se debe a la interpretación de las configuraciones de algunos nombres ${ }^{14}$ (la ae de TELEVISION, la ëa de MONEDA, la ó de LAMPARA, escritos en S.E.A.) como configuraciones clasificatorias. El hecho de emplear una configuración clasificatoria o la misma configuración del nombre es en cierto modo análogo a lo que ocurría en las LOs con la incorporación del nombre y la afijación de un morfema que lo representa. Pero ya vemos que la analogía termina allí. Pues lo que en las LOs son extensiones morfológicas de un verbo, en la LSE es sustitución morfológica de un componente fonológico, la configuración, del verbo, del adjetivo o del locativo, en sus formas léxicas habituales sin clasificación ${ }^{15}$.

Por otra parte, la clasificación se da especialmente cuando la forma léxica correspondiente al verbo, adjetivo o locativo o no existe (por ejemplo no hay forma léxica para caer, poner, etc) o tiene una configuración que se siente no como universal, sino como clasificatoria (por ejemplo el adjetivo BLANDO tiene forma léxica, pero se modifica por ejemplo para signar SUELO BLANDO).

En la LSE, por consiguiente, la clasificación de un nombre en el adjetivo, el verbo o el locativo referidos a ese nombre se produce de forma abrumadora por introflexión. Esta introflexión afecta a la configuración del signo receptor (si la tiene) que cambia esa configuración por la del clasificador del nombre o por la del nombre mismo. En sentido estricto, pues, los clasificadores predicativos no se realizan en la LSE por incorporación del nombre.

14 Se ha sugerido, a partir de Kegl y Scheley (1986) que todos los signos del lexicon de la ASL incluyen clasificadores o derivan de un predicado clasificatorio.

15 En la LSE se dan otros casos de supleción por introfiexión, que no deben entender como clasificadores, como es el caso de la incorporación del número a nombres temporales (día, semana, mes, etc) o a verbos. Es importante no confundir esta incorporación con la clasificación. 
Este procedimiento es, desde mi punto de vista, perfectamente congruente con la tipología, salvo que en los estudios tipológicos no se suele extender lo predicativo al ámbito del SN, concretamente a los complementos adjetivos y locativos, a no ser que funcionen predicativamente; y salvo que el término supleción debería especializarse en raíces inanalizables.

El tercer tipo datado tipológicamente, el de los clasificadores afijados, no ha sido tampoco tratado, hasta donde sé, en las LSs., sin duda porque en la noción misma de afijo está involucrada una concepción del orden fonológico de la palabra o del signo. Para terminar, vamos a proponer la existencia de clasificadores afijales en la LSE, a partir de un modelo fonológico del signo que podemos llamar radicalmente secuencial pero que, de hecho, se basa en evidencias perceptivas que, al menos para el caso de estos clasificadores, pueden resultar esclarecedoras.

\section{Los clasificadores afijales en la LSE}

Una de las características fonológicas de las LSs es que cuentan con dos articuladores manuales, las dos manos. Que se trata de un componente fonológico lo demuestran los numerosos signos bimanuales, ya sea por simetría o cuasi-simetría de las dos manos (como en PIEDRA, VIAJES, VACACIONES, etc) o porque la mano pasiva actúa como lugar fonológico de la mano activa en el signo, adoptando (a diferencia de otros lugares del cuerpo) una configuración y una orientación determinadas (como en CARÁCTER, COSA, ESCRIBIR, etc.). No vamos a entrar en los procesos morfológicos en los que puede entrar en general la mano pasiva, sino sólo en su función de clasificador, como es el caso de mirar una manzana, golpear una botella, echar aceite en una tostada, y otros muchos ${ }^{16}$.

En estas construcciones la mano pasiva representa el objeto de la acción expresada por la mano activa, a través de un clasificador de ese objeto o, en su defecto, de su misma configuración, como hemos visto que sucedía con los clasificadores verbales. Pero fijémonos en que en estos casos la configuración del verbo es la habitual, no hay introflexión: VER, GOLPEAR o ECHAR ACEITE se realizan íntegramente. Sólo el lugar de realización del signo y la orientación se especifican en relación a la mano pasiva.

Para comprender el valor morfológico de la mano pasiva en estos ejemplos tan frecuentes en la LSE debemos tener muy en cuenta esta diferencia con otros clasificadores, pero sobre todo que, como ya hemos comentado, el componente fonológico o fonema de lugar de los signos es secuencial y procesualmente anterior a la especificación del resto de fonemas, por lo que puede ser considerado como el ataque silábico habitual; esta anterioridad se evidencia especialmente en los signos bimanuales de los tipos que hemos referido, con clasificador (mirar una manzana) o sin él (CARÁCTER), en los que claramente es la mano activa la que se desplaza hacia la pasiva, que se fija previamente. Por eso, en nuestro sistema de escritura (Herrero, 2003), el signo comienza por los fonemas de lugar. Por ejemplo, el signo MIRAR, que se signa delante de los ojos, se escribe v.eimeu, con el símbolo correspondiente a este lugar y a la falta de contacto (v.), para seguir con la configuración (ei) y la orientación

16 Del mismo modo que algunos signos parecen incorporar una configuración de la mano activa clasificatoria, o al menos motivada, en signos bimanuales de mano pasiva ésta parece también clasificatoria, como es el caso de ESCRIBIR. 
(meu); en este signo no hay movimiento fonológico ${ }^{17}$ ¿Cómo escribiríamos entonces mirar una manzana cuando el objeto, la manzana, está representada por la mano pasiva? Haríamos preceder el signo MIRAR del signo de la mano izquierda (o de su clasificador), en este caso del signo MANZANA (abso'áma), para formar el signo bimanual:

abso'ma-v.eimeu

manzana-mirar

mirar una manzana

Lo mismo ocurriría con los otros ejemplos. Aunque las dos manos podrían emplearse con una gran riqueza de valores morfológicos y/o de plasticidad (Lidddell, 2003), aquí estamos ante formas sistemáticas, similares a la que se expresan en signos simples. Esos signos comienzan con su mano pasiva, como lugar de realización del signo. En los signos que estamos tratando, y dentro de la construcción predicativa con objeto directo, la mano pasiva se añade al signo verbal, como un afijo. Es lo que sucede en mirar una manzana, pero también en mirar una fotografia, mirar un libro, etc.

Creo que el paradigma de estas construcciones muestra claramente su congruencia con los casos de clasificadores verbales afijados que la tipología ha constatado.

\section{Conclusiones}

Hemos presentado, de acuerdo con los últimos estudios tipológicos sobre clasificadores, un breve panorama de la morfología de la clasificación nominal en la LSE. Esta lengua de signos presenta clasificadores nominales (que hemos preferido llamar nombres clasificatorios), que son morfemas libres léxicamente activos en otros contextos, y clasificadores verbales -pero también adjetivales y locativos-, o simplemente configuraciones clasificatorias, que son morfemas introflexivos, y por tanto ligados. En el caso de los nombres clasificatorios, hemos dado pruebas de su diferencia respecto de construcciones sinónimas como compuestos, complementos y derivados, y hemos relacionado clasificación e incorporación. En el caso de los clasificadores (verbales, adjetivales, locativos) hemos intentado demostrar su carácter morfológicamente introflexivo, y hemos deslindado el caso de los clasificadores de mano pasiva que consideramos morfológicamente como afijos de la categoría léxica respectiva.

En cuanto a la influencia que el modo de representación visual de las lenguas de signos puede tener en la clasificación, creemos que se limita a la expresión, no al sistema mismo de clasificación, que es tipológicamente congruente con los sistemas de clasificación de lenguas orales. El modo visual permite la extensión de lo sistemático más allá de los estrictamente lingüístico, sometiendo configuraciones y movimientos a una plasticidad que puede llegar a esconder su base sistemática.

17 Brentari (2002: 44): "regarding minimal word constraints, no sign is well formed unless it has a movement of some type". Esta idea, que está en la base de la concepción nuclear del Movimiento, inspira los modelos fonológicos del signo de S. Liddell y de D. Brentari. 


\section{Referencias bibliográficas}

Aikhenvald, Alexandra Y. (2000): Classifiers. A Typology of Noum Categorization Devices. Oxford, Oxford University Press.

Aikenvald, Alexandra Y. (2003): "Classifiers in Spoken and in Signed Languages: How to Know More". Perspectives on Classifier Constructions in Sign Languages ed. por Karen Emmorey. Mahwah, N.J., Lawrence Erlbaum Associates. págs. 87-90.

Allan k. (1977): "Classifiers". Language, 53, págs. 284-310.

Bergman, Brita \& Lars Wallin (2001): "The discourse function of noum classifiers in Swedish Sign Language". Signed Languages: Discoveries from International Research ed. por V.Dively, M.Metzger, S.Taub \& A.M.Baer. Washington DC, Gallaudet University Press, págs. 45-60.

Bergman, Brita \& Lars Wallin (2003): "Noum and Verbal Classifiers in Swedish Sign Language". Perspectives on Classifier Constructions in Sign Languages ed. por Karen Emmorey. Mahwah,

N.J., Lawrence Erlbaum Associates, págs. 35-51.

Brentari, Diane (2002): "Modality differences in sign language phonology and morphophonemics".

En Meier, Richard P.; Kearsy Cormier and David Quinto- Mozos (eds.), Modality and structure in signed and spoken languages. Cambridge, Cambridge University Press, págs. 35-64.

Cogill-Koez, D. (2000a): "A model of signed language 'classifier predicates' as template visual representation". Sign language and Linguistics, 3:2, págs. 209-236.

Cogill-Koez, D. (2000b): "Do Athapaskan language provide a precedent for signed language "classifier predicates'?". Sign language and Linguistics, 3:2, págs. 257-259.

Corbett, G. (1991): Gender. Cambridge, Cambridge University Press.

Craig, C.G. (ed) (1986): Noum Classes and Categorization: Proceedings of a Symposium on Categorization and Noum Classification (1983). Amsterdam, John Benjamins.

Croft, William (1994): "Semantic Universals in Classifier Systems". En Word 45, págs. 145-171.

Dixon, R.M.W. (1968): Noum Classes. En Lingua 21, págs. 104-125.

Dixon, R.M.W. (1986): "Noum Clases and Noum Classification in Typological Perspective". En Craig (ed.), págs. 105-12

Emmorey, Karen (ed.) (2003): Perspectives on Classifier Constructions in Sign Languages. Mahwah, New Jersey, Lawrence Erlbaum Associates, Pub.

Engberg-Pedersen, E. y A. Pedersen (1985): "Proforms in Danish Sign Language: Their use in figurative signing". En William Stokoe y V. Volterra (eds.) SLR'83: Proceedings of the III International Symposium on Sign Language research. Silver spring, MD. \& Rome, Linstok Press \& Instituto di Psicologia CNR.

Engberg-Pedersen, E. (1989): "Proformes en morphologie, syntax et discourse". En S. Quertinmont (ed.) Études Européenes en langues de signes. Bruselas, Irsa, págs. 35-52.

Frishberg, Nancy (1972): "Navajo object markers and the Great Chain of Being". En J.Kimball (ed.) Syntax and Semantics, Volume I. Nueva York, Seminar Press.

Frishberg, Nancy (1975): "Arbitrariness adn iconicity: Historical change in American Sign Language". Language, 51 , págs. 676-710.

Greenberg, Joseph. H. (1972): "Numeral Classifiers and Sustantival Number: Problems in the Genesios Type". En Working Papers in Language Universals. Reimpreso en K. Denning y S.Kemmer (eds.) On Language: Selected Writings of Joseph H. Greenberg. Stanford, Stanford University Press, 1990, págs. 241-270.

Grinevald, Colette (2000): "A morphosyntactic typology of classifiers". En G.Senft (ed.) Systems of noninal classification. Cambridge, Cambridge University Press.

Grineval, Colette (2203): "Classifier Systems in the Context of a Typology of Noum Classification". En Karen Emmorey (ed.), págs. 91-110.

Hervás, Lorenzo de (1795): Escuela española de sordomudos o Arte para enseñarles a escribir y hablar el idioma español. 2 Tomos. Madrid, Imprenta Real. 
Herrero, Angel (2003): Escritura alfabética de la Lengua de Signos Española. Once lecciones. Alicante, Publicaciones de la Universidad de Alicante.

Herrero, Angel (2004): "A practical writing of sign languages". (in press.).Lisboa: $4^{\text {th }}$ International Conference on Language Resources and Evaluation (LREC 2004).

Herrero, Angel \& Ventura Salazar (2004a): (en press.) "Non-verbal predicability and copula support rule in spanish sign language". En Groot, Gasper de y Kees Hengeveld (eds.), Morphosyntactic expression in Functional Grammar. Berlin, Mouton de Gruyer.

Kegl, J.A. \& Ronnie Willbur (1976): "When does structure stop and style begin? Sytax, morphology, and phonology vs. stylistic variation in American Sign Language". En C.Mufwenw y S.Steever (eds.) Papers from the Twelfth Regional Meeting.Chicago Linguistgics Society. Chicago,University of Chicago Press., págs. 376-396.

Kegl, J.A. \&S. Schley (1986): "When is a classifier no longer a classifier?" Proceeding of the Annual Meeting of the Berkeley Linguisticsn Society, 12, págs. 425-441.

Liddell, Scott K. (2003): "Sources of Meaning in ASL Classifier Predicates". En Karen Emmorey (ed.), págs.199-220.

Meier, Richard P.; Kearsy Cormier \& David Quinto-Mozos (2002): Modality and structure in signed and spoken languages. Cambridge, Cambridge University Press.

Moreno Cabrera, Juan Carlos (1997): Introducción a la lingüística. Énfoque tipológico y universalista. Madrid, Síntesis.

Royen, G. (1929): Die nominalen Klassifikations-Systeme in den Sprachen der Erde. Viena, Anthropos

Sapir, Edward (1921): Language. An Introduction to the Study of Speeech. Nueva York: Harcourt, Brace and Co., Inc. Ed. en español en México D.F Fondo de Cultura Económica, 1974.

Sapir, Edwuard (1932): Two Navajo Puns. En Language 8, págs. 217-219.

Schembri, Adam (2003): "Rethinking 'Classifiers' in Signed Languages". En Emmorey, Karen (ed), págs. 3-34.

Simone, Raffaele (1993): Fundamentos de lingüistica. Barcelona, Ariel.

Supalla, Ted (1978): "Morphology of verbs of motion and location in American Sign Language". En FR.Caccamise y D. Kicks (eds.) American Sign Language in a bilingual, bicultural context: Proceedings of the Second National Symposium on Sign Language Research ande Teaching. Coronado, CAQ, National Association of the Deaf, págs. 27-46.

Supalla, Ted (1986): "The classifier system in Americal Sign Language". En C. Craig (ed.), págs. 181-214.

Supalla, Ted (2003): "Revisiting Visual Analogy in ASL Classifier Predicates". En Karen Emmorey (ed.), págs. 249-258.

Wallin, Lars (1996): Polysynthetic signs in Swedish Sign Language. Estocolmo, University of Stockholm.

Worsley, P.M. (1954): "Noum-classification in Australian and Bantu: Formal o Semantic?", Oceania 24, págs. 275-288. 